\title{
Entropy, Biological Evolution and the Psychological Arrow of Time
}

\author{
Torsten Heinrich'1, Benjamin Knopp ${ }^{2}$, Heinrich Päs ${ }^{3}$ \\ ${ }^{1}$ Institute for Institutional and Innovation Economics, University of Bremen, Bremen, Germany \\ ${ }^{2}$ Fachbereich Physik, AG Neurophysik, Philipps-Universität Marburg, Marburg, Germany \\ ${ }^{3}$ Fakultät für Physik, Technische Universität Dortmund, Dortmund, Germany \\ Email: torsten.heinrich@uni-bremen.de, benjamin.knopp@physik.uni-marburg.de, \\ heinrich.paes@tu-dortmund.de
}

Received 28 October 2015; accepted 26 January 2016; published 29 January 2016

Copyright (C) 2016 by authors and Scientific Research Publishing Inc.

This work is licensed under the Creative Commons Attribution International License (CC BY).

http://creativecommons.org/licenses/by/4.0/

c) (i) Dpen Access

\begin{abstract}
We argue that in Universes where future and past differ only by the entropy content a psychological arrow of time pointing in the direction of entropy increase can arise from natural selection in biological evolution. We show that this effect can be demonstrated in very simple toy computer simulations of evolution in an entropy increasing or decreasing environment.
\end{abstract}

Keywords

Arrow of Time, Time Asymmetry, Entropy, Evolution

\section{Introduction}

While the laws of classical physics are perfectly time-symmetric, i.e. causality works equally well forward and backward in time both daily life and the microcosm governed by quantum laws are time-asymmetric (excellent monographs on the nature of time include [1]-[3] and the collections of essays [4] [5]). This asymmetry manifests itself in various arrows of time:

- The thermodynamical arrow of time, as provided by the second law of thermodynamics: the entropy in a closed system always increases or remains constant and never decreases. For example, intact cups can fall off the table and splatter in thousand pieces, releasing their potential energy as heat radiation, while cup pieces on the floor never cool down their environment and use this energy to unbreak and jump onto the table. Of course for a system to undergo entropy increase it has to be out of equilibrium in the first place, i.e. starting from a low entropy state.

- The cosmological arrow of time [6] [7]: the Universe Hubble expands as time proceeds. 
- The radiation arrow of time: (electromagnetic) waves propagate retarded, i.e. outward from the source.

- The quantum mechanical arrow of time: pure states "collapse” or decohere (depending on one's preferred interpretation of quantum mechanics) into mixed states but mixed states never evolve into pure states.

- The CP asymmetry: weak interaction processes in particle physics proceed with different rates if the direction of time is reversed.

- The biological arrow of time: living creatures die but corpses do not revive.

- The psychological arrow of time: it is possible remember the past but not to "pre-member" the future.

It is interesting to note, though, that not all of these arrows of time are independent. Most obvious, the biological arrow of time is just another example of entropy increase. After all, entropy in its statistical interpretation is nothing but a measure of how many microstates correspond to a given macrostate, and thereby a measure of how probable this macrostate is: there are simply many more possible ways for a cup to be broken than to be unbroken, and many more ways for being dead than for being alive (almost all organs intact, working and in their right places).

Beyond that, in modern physics it actually becomes more and more common to assume that all arrows of time are just different manifestations of entropy increase:

- The cosmological expansion may be intimately related to the thermodynamical arrow of time by actually defining the direction in which entropy would increase by providing a low entropy state at the beginning of time. In such scenarios time would start to run backwards if the Universe would recollapse. Alternatively, the cosmological arrow may be no arrow at all, as a Universe shrinking with time could be possible as well, given different initial conditions.

- The radiation arrow of time can result from boundary conditions as well.

- If the quantum mechanical arrow of time is due to decoherence [8] as in the Everett-Many-Worlds interpretation [9] it is simply a consequence of entropy increase once a quantum system is entangled with its environment in a measurement process. This results from the fact that the microstates of the environment are not accessible to the observer.

- While the CP symmetry is violated in weak interaction processes CPT remains conserved and may be interpreted as the correct operation of time inversion.

The notion that time is an illusion, that it is nothing but entropy increase after all, is further supported by the fact that the Wheeler-de-Wit equation [10]

$$
\hat{H}|\psi\rangle=0
$$

for the quantum mechanical wave function of the Universe [11] in canonical quantum gravity models does not include any time coordinate. In quantum cosmology, time may thus rather come into being as an emergent phenomenon when the transition to a semi-classical description of Nature is made, i.e. quantum fields evolving in a background of classical gravity, since by separating out gravity as a classical background of spacetime a macrostate is defined corresponding to many possible microstates in the full quantum gravity description (see e.g. the discussions in [12]-[17]).

In the following, we thus adopt the hypothesis that entropy increase is the only manifestation of time. It should be stressed that this assumption makes time a psychological phenomenon. After all the entropy of a given macrostate depends on its definition in terms of microstates, a property that cannot be derived from the fundamental laws of physics. If this hypothesis is correct, however, the most obvious manifestation of time becomes the most puzzling one: the psychological arrow of time- the fact that we remember the past but do not "premember" the future.

More concrete, if the entropy budget is the only difference between past and future one would expect that building up memory would correspond to entropy increase. Quite the opposite is true, though. By organizing our brains in a way to store memory the unordered high entropy state of the brain evolves into an organized low entropy state [22]-[25] $]^{1}$. Of course this is possible as the brain is an open system, and the process of building up memory and reducing entropy locally inside the brain can proceed at the cost of increasing entropy in the environment. But this consideration seems to suggest that if entropy increase is the only manifestation of time there

\footnotetext{
${ }^{1}$ Note however that there exists a controversial exchange of arguments between Hawking [18], Hartle [19], Schulman [20] and Maroney [21] on whether the psychological arrow can be explained as a special case of a computational arrow of time which has to proceed necessarily in the direction of entropy increase, based on the argument that memory requires the erasure of record beforehand [22]. We consider this controversy as unsettled.
} 
would be no reason why we shouldn’t pre-member the future, thereby naturally growing entropy in our brain instead of forcing a local entropy decrease by using up free energy.

In the following, we will follow this line of reasoning and show that in the case the fundamental laws of physics would allow to pre-member the future, natural selection in the course of biological evolution could eliminate individuals developing memory of the future.

There exist at least two classical explanations for the psychological arrow of time in the literature which are related to but different from the approach adopted here (compare the discussions of Zeh [1], Albert [3] and Carroll [26] as well as [27] [28]): First, one can argue that memory is just a special case of a document of the past. Such documents are more strongly correlated with events in the past than with events in the future, since documents of the future could originate with a higher probability from a statistical fluctuation (we will come back to this argument in the discussion section). Next, information is typically transmitted by (electromagnetic) radiation and thus subject to the radiation arrow of time which is a consequence of boundary conditions in an entropy increasing environment. One may argue that these explanations should be considered more fundamental than any explanation relying on the utility of memory as they concern the basic concept and origin of memory itself, but this is not necessarily the case. Recently, Rovelli argued for example that the coarse-graining hypothesis in defining a macrostate which is necessary to calculate its entropy is a consequence of the interaction creatures "living in time" have with the Universe [29] [30]. Tegmark makes a similar argument when he supposes that the emergence of consciousness and the emergence of time may be related [31]. In this sense we adopt the Archimedean or atemporal standpoint demanded by Price [2] who argued that that the asymmetry of causation is anthropocentric in origin. In the remainder of the paper we will thus focus on the superior predictive power of memory of the past as compared to memory of the future as a possible cause for the psychological arrow of time.

The basic argument here is simple. Memory has evolved in the course of biological evolution for only one reason: namely, in order to increase the fitness of an individual in a situation in the present. In its most basic form, memory is helpful by providing a set of initial conditions which allow to calculate (or at least estimate) the behavior of enemies, prey or prospective sex partners in the present. The fundamental laws of physics which govern this behavior are classical: they work as well forward as backward. However, in order to calculate a process in the present from initial conditions in the future one typically has to deal with a higher entropy state than by calculating the process from initial data in the past: For an illustrative example, just imagine you try to avoid being hit by a rock. You see the position of the rock on top of a hill and you can easily estimate how it will fall to the ground, where it disintegrates (see Figure 1, left panel). The problem reduces to solving a very simple differential equation. If you want to achieve the same goal by relying on initial data in the future, you have to know the positions of all constituents of the disintegrated rock, and calculate how and where exactly they integrate to form the rock flying up onto the hill (Figure 1, right panel). There are far more initial conditions to memorize, and the problem now transformed into a complicated multi-dimensional set of coupled differential equations.
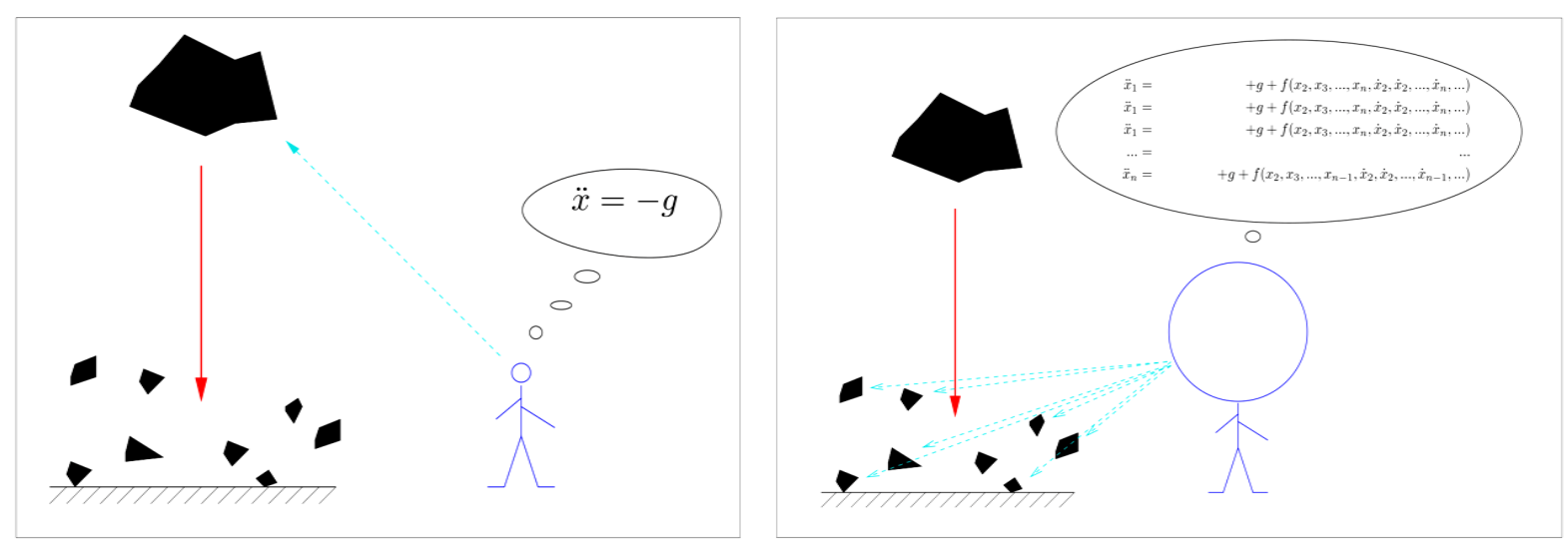

Figure 1. Illustration of an individual escaping a threat by processing memory stored about the past (left panel) and escaping the same threat by processing memory stored about the future (right panel). 
As entropy is a measure of the information required to describe the microstate realized in a given macrostate, an individual would have to store and process more information. And, depending on the situation it wants to manage, the amount of information may not be a factor of few, but will typically grow exponentially the farther your memory lasts into the future. Storing and processing information requires storage, and-metaphorically oversimplified - the more storage one needs the larger one's brain has to be (see e.g. the comparison of various animal brain sizes in [32]). At some point (and this point will come pretty soon) such a large brain will make the individual's ability to move and react (the only reason to develop memory in the first place) more and more difficult and finally impossible. Such species will never prevail or even develop in the course of evolution that favors agility and swift decision-making. If we consider the psychological arrow of time as a possible consequence of biological evolution though, we run into the difficulty that we typically understand evolution as a process in time while we now use it to establish an (at least seemingly) very fundamental property of time itself. The question is thus how we understand evolution and survival in a time-symmetric Universe.

As we point out here, though, it is not too difficult at all to imagine such a scenario. For sake of simplicity we model evolution by a computer simulation. A very simple realization would study two contrasting scenarios with one in which the lifeforms remember the past while in the other they pre-member the future ${ }^{2}$.

\section{A Simple Toy Model}

In order to address the question whether remembering populations have a superior fitness compared to premembering populations we have developed a very simple simulation as a toy model for artificial life and evolution of a population in a hostile environment.

The individuals of a given population are modeled as agents moving in a two-dimensional grid. A hostile environment is simulated by adding falling rocks with a given mass which move predictably according to the laws of classical physics. At the boundary of the grid the rocks disintegrate into two rocks with half of the mass of the original rock. The exact rules for collisions with the boundaries are depicted in Figure 2. Once the mass of a rock reaches the value of 0.5 it constitutes no threat for individuals anymore and is removed from the simulation. New rocks are generated randomly with a given probability in order to ensure a stable environment with a more or less constant rock density oscillating around an average value. The rocks are responsible for both selection and for the irreversibility of processes in the environment which allows to define the direction of time as the direction of increasing entropy. The evolution of the rock environment is being recorded first and can subsequently be run both in forward and backward direction. Finally populations of individuals can be placed in either the forward or the backward running environment and their probabilities for extinction or survival can be compared.

The individuals possess a given number of lives which gets reduced by the mass value of a rock when they get hit. When the number of lives is reduced to zero the individual "dies" and gets removed from the simulation. The individuals reproduce asexually by reduplication with a defined probability $p_{r}$ with each time step. Furthermore the individuals scan the adjacent and next-to-adjacent cells (a two-cell Moore-neighborhood) of their respective position and avoid a threatening rock by moving out of its way once it is detected. The limited memory of an individual is modeled by allowing individuals to find and avoid only one single rock - whenever two or more rocks threaten the individual it is at danger of being hit. If no rock is observed, the individuals move or rest randomly. For a screen shot of the simulation, see Figure 3.

\section{Results}

In order to compare the probabilities for extinction or survival of past-remembering and future-premembering populations a population of individuals is situated either in the forward or backward running environment of scattering rocks and evolved according to the simple rules discussed above. As a consequence, populations can remain stable over a given time span, die out or explode. For high $p_{r}$ or a low number of stones, populations are more likely to explode, for low $p_{r}$ or high numbers of stones, they are more likely to become extinct. In between there is a critical region. Due to statistical influences induced by the random creation of rocks and random replication of individuals as well as their random initial positions the simulation may result in all possible outcomes even for a single set of initial conditions within the critical region. To study the exact differences

\footnotetext{
${ }^{2}$ Note that the model does not really rely on any "flow of time" at all (as demanded by Price for an atemporal perspective [2]) but can be considered as different states linked by a causal connection (the physical laws) without explicit reference to time at all.
} 

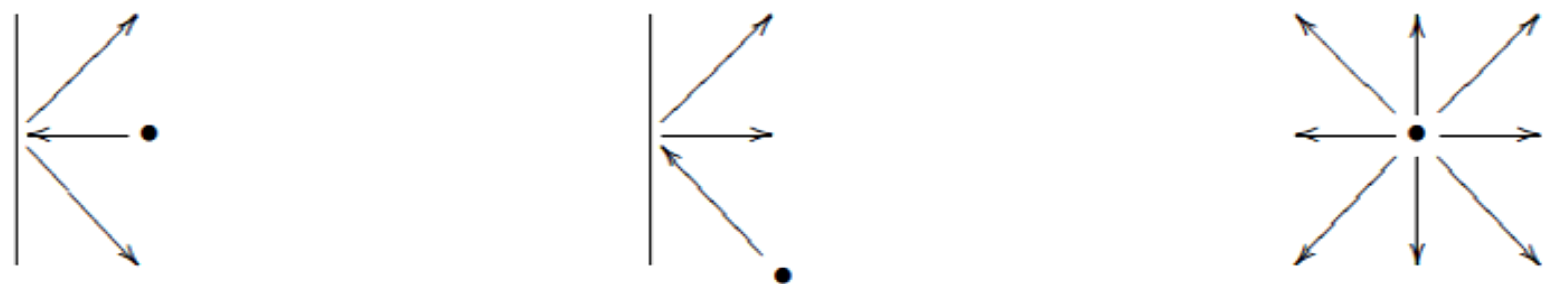

Figure 2. Rock movements and disintegration on the walls of the grid.

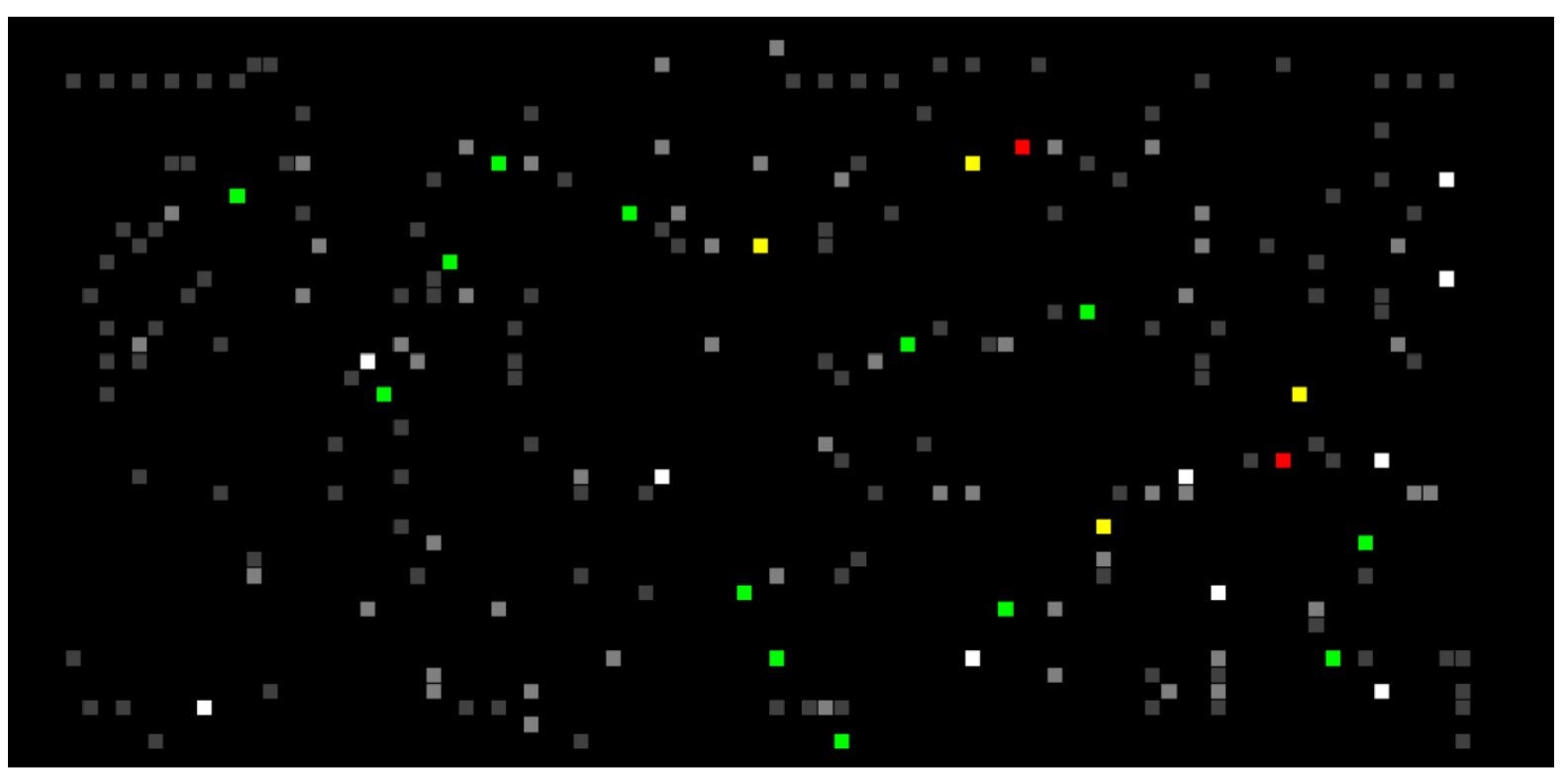

Figure 3. Screen shot of the simulation. Individuals are depicted as green (3 lives), yellow (2 lives) or red (1 live) cell, rocks are depicted as white (mass 4), light grey (mass 2) or dark grey (mass 1) cells.

between the two settings we investigate (past remembering and future pre-membering lifeforms), we thus performed a statistical analysis, defining the value $p_{1 / 2}$ as the reproduction probability $p_{r}$ for which more than half of the simulations result in a stable population and survive a given running time of 5000 iterations (for an example of a stable population over a certain time span see Figure 4$)^{3}$.

The result is shown in Figure 5 where the percentage of stable populations is plotted as a function of $p_{r}$. Here red and green crosses correspond to past-remembering and future-premembering populations, respectively and the respective values for $p_{1 / 2}$ can be read off as a result of linear extrapolations to the 50\% survival rate.

For populations in an entropy increasing environment the reproduction rate necessary for a stable population is $(3.7 \pm 0.002) \times 10^{-2}$ while for populations in an entropy-decreasing environment the necessary rate is $(3.9 \pm$ $0.009) \times 10^{-2},$. .

\section{Discussion}

At this point a few remarks are in order:

\footnotetext{
${ }^{3}$ Note that $p_{1 / 2}$ may vary with the length of the simulation, particularly for smaller numbers of iterations. For the number of iterations used here (5000), this effect is greatly reduced. One might consider using the threshold for which the probability for the population to grow or to decline would be equal. However, this would result in many simulation runs with—potentially exponentially—expanding numbers of agents which, in turn, would increase the number of computation operations per iteration accordingly leading to requirements in computation power that is by magnitudes larger than what we can employ.

${ }^{4}$ This difference and its statistical significance seem to be stable across different parameter settings (while the values themselves are of course sensitive to parameter changes). The parameter setting we used includes an initial number of agents of 30 , an initial number of rocks of 10 with an initial mass of 3 each and a probability of 0.4 that a new rock is injected in each iteration on a rectangular grid of $43 \times 23$ cells. The simulation runs had a length of 5000 iterations; the simulations for each candidate $p_{r}$ were repeated 100 times in order to obtain a good estimate for the corresponding survival rate.
} 


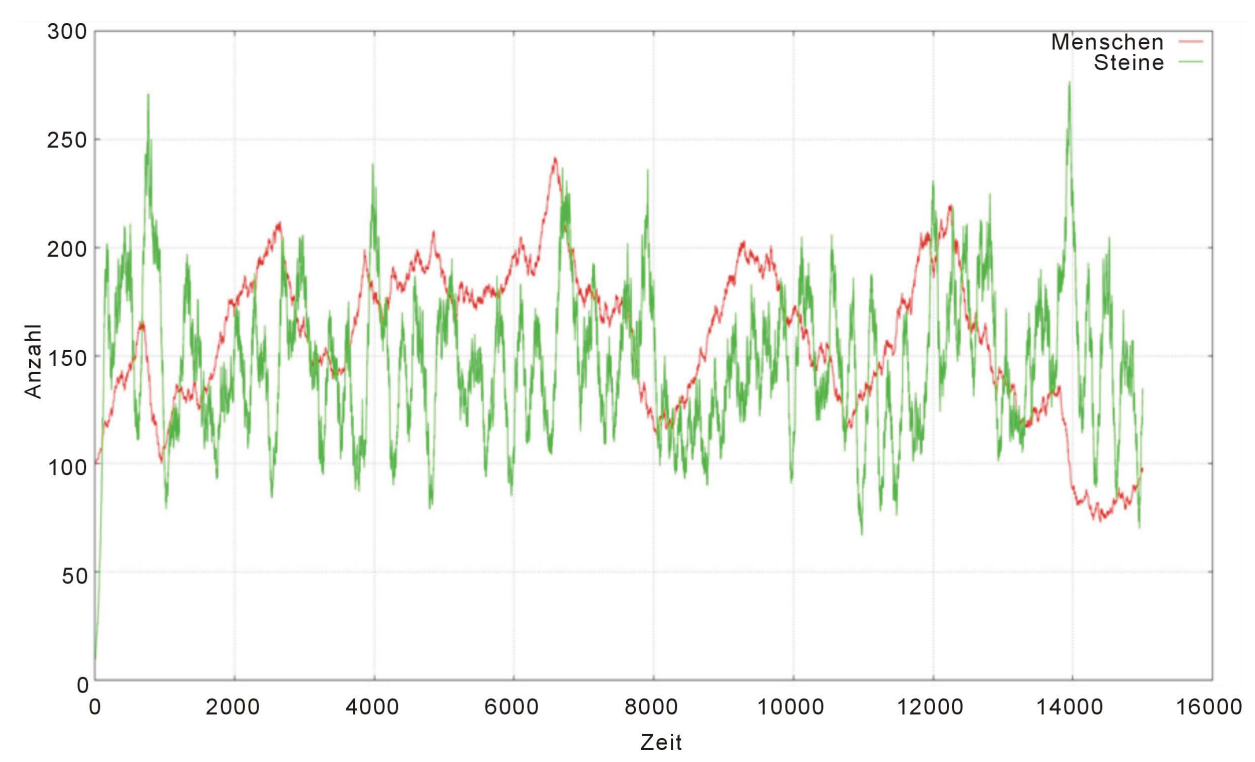

Figure 4. Stable population of individuals (red) in comparison to the number of rocks in the grid (green) as a function of running time/iterations.

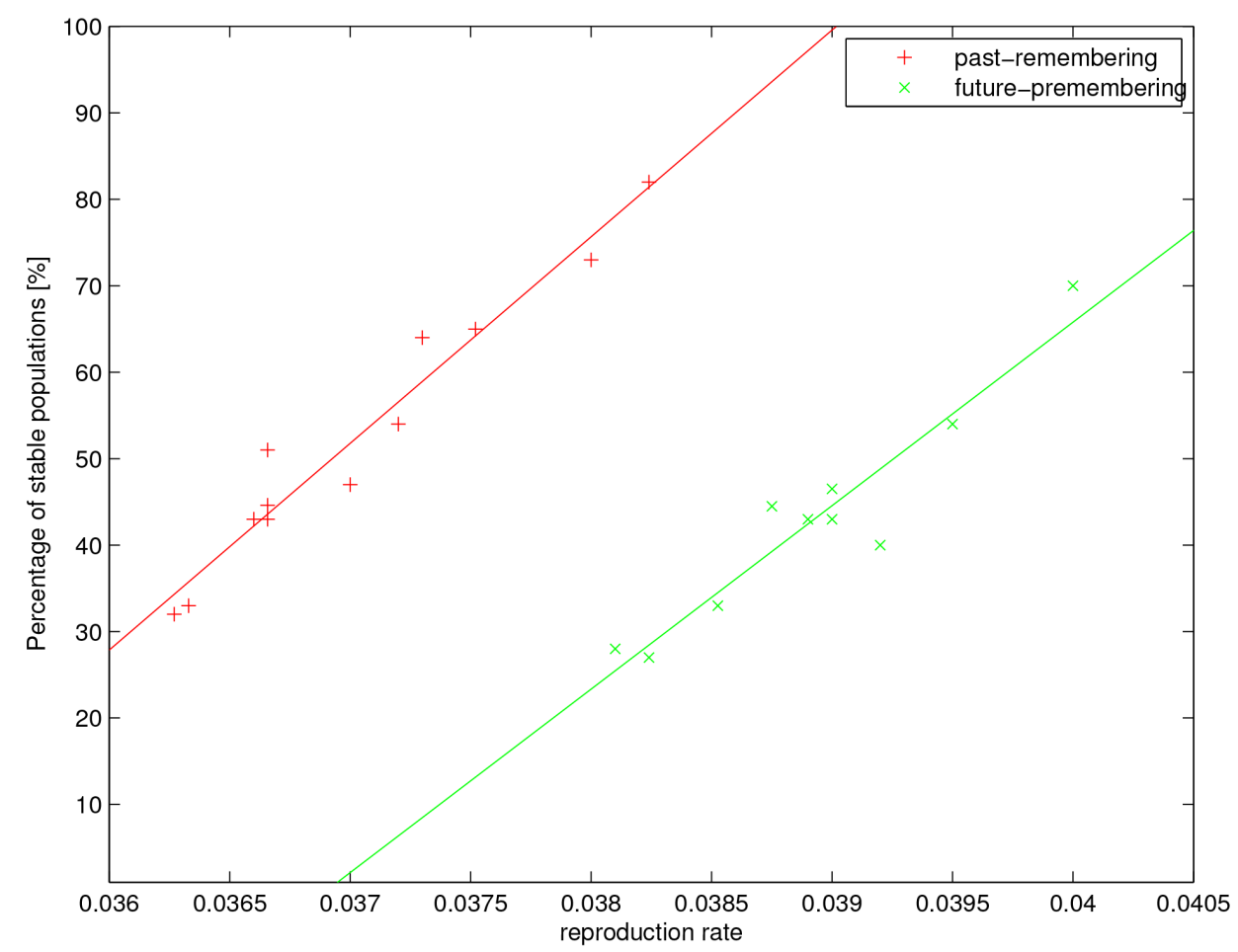

Figure 5. Percentage of stable populations as a function of the reproduction rate $p_{r}$ for past remembering (red crosses, upper fit) and future-premembering populations (green crosses, lower fit). The mean reproduction rate with a $50 \%$ survival chance $p_{1 / 2}$ is obtained by linear extrapolation.

First, we chose to adopt in our simulation what may be called a psychological perspective, as it corresponds to the subjective perception of the pre-membering population (starting with birth and ending with death). That means, if we define the direction of time as the direction of increasing entropy, our pre-membering populations are not really living forward in time with a memory of the future, but rather live entirely backwards in time. We 
chose the psychological perspective here in order to have a simple, model that allows to compare re-membering and pre-membering populations in a transparent way. It could be argued though that the former, biological perspective is more realistic as life typically is understood as a process evolving in the direction of entropy increase. As the mechanisms responsible for the time asymmetry are the same in both cases we do not expect a qualitative change in our results, if the biological perspective is chosen instead.

Such a biological perspective has been employed in a simulation performed by Zeissner [33], where a previously recorded environment could be accessed by remembering and pre-membering agents at times $t_{0}-1$ and $t_{0}+1$, respectively. It was noted that under these conditions the pre-membering population could access the same information as the remembering population, albeit two time steps earlier. This results in an advantage of the pre-membering population which competes with the disadvantage of the minor predictive power of the "rediction" process. Which effect dominates depends on the actual situation but it seems clear that for realistic environments the superior predictive power of past-memory will be more important.

Second, in our simulation the cost of information storage in the memory is not modeled. We rather compared the capabilities of populations to survive in entropy increasing and entropy decreasing environments assuming they have the same skills in information processing. This can be motivated by the fact that the brain is an open system, and that recording memory in our brains works against the external entropy increase anyway, but it leaves open the question whether backward living lifeforms can store information at no or even negative cost. A psychological arrow of time requires to sustain the physical basis for memory storage (a "big brain") over a large time period in parallel to the biological arrow of time corresponding to entropy increase though we consider any possible amount of free energy generation by storage of pre-membrance as negligible.

Third, as discussed previously and related to the point above, it has been pointed out by Zeh [1], Albert [3], Carroll [26] and others, that if memory can be generated automatically at no cost in free energy, "false memory" in the sense of information stored inside the brain which has no correspondence to real information about the environment is more probable and thus such memory will be less reliable. This effect will strengthen the evolutionary argument for back-in-time remembrance but is more difficult to simulate and probably less important than the simple argument advocated here.

\section{Conclusions}

In this paper, we were studying a very simple toy model modeling biological evolution of past-remembering and future-premembering populations in order to compare the probabilities for extinction or survival of these populations. While this work obviously does not prove that the real psychological arrow of time is a product of biological evolution and relies on many assumptions, is extremely simple and custom built, it does demonstrate, though, that-if the fundamental laws of physics would allow to pre-member the future-natural selection could be sufficient to eliminate this skill.

A critical argument often raised is that the simulation compares only backward memory with future memory, but not with hybrid memory concepts, typically phrased as "a limited knowledge of the future could be very helpful”. This argument misses the fact that any kind of memory is costly. This is the reason why we don't remember the entire past but only an apparently most significant subset of it in the first place. Taking the extreme case, already by remembering the entire past a deterministic Universe would allow Laplace's demon to calculate the entire future with absolute precision at a lower or equal cost than by pre-membering the future. This argument suggests that any useful amount of memory of the future would be too costly to prevail in a natural selection process.

Finally, a well-motivated question is how to test these ideas. More realistic computer simulations beyond the simple toy model described here would definitely help. More convincing, of course, would be to probe the idea in an actual biological experiment. In principle one could study for example the evolution of a population of bacteria in a local environment where entropy is always decreasing. If the argument advocated in this work is correct, it should be possible for such a population to develop memory of the future, but it is totally unclear whether such a development could occur fast enough to be observable and if the population could survive long enough under such conditions to evolve sufficiently ${ }^{5}$. To discuss how realistic such a setup is thus beyond the scope of this work.

\footnotetext{
${ }^{5}$ Note that "memory" here does not refer to the genome of these bacteria but rather to less complex information processing mechanisms the bacteria rely on, for instance the presence or absence of chemical substances in the bacterium which plays a part in governing its movement in species that are able to actively move through bacterial gliding.
} 
In summary, we thus consider this work as a proof of principle that it is possible to discuss a possible origin of the psychological arrow of time from natural selection in a meaningful scientific way.

\section{Acknowledgements}

HP would like to thank Peter Schupp for inspiring his research in this direction by arguing the psychological arrow of time may actually result from psychology, as well as Marc Brown, Sean Carroll, Lou Clavelli, Frank Deppisch, Carlo Rovelli, Ketil Soerensen, Rüdiger Vaas, Sinan Zeissner, Heinz-Dieter Zeh for useful discussions during various stages of this work. All remaining errors are our own.

\section{References}

[1] Zeh, H.D. (1991) The Physical Basis of the Direction of Time. Springer, Berlin. http://dx.doi.org/10.1007/978-3-662-02759-2

[2] Price, H. (1996) Time’s Arrow and Archimedes’ Point. Oxford University Press, New York.

[3] Albert, D.Z. (2003) Time and Change. Harvard University Press, Cambridge.

[4] Halliwell, J.J., Perez-Mercader, J. and Zurek, W.H. (1994) Physical Origins of time Asymmetry. Proceedings, NATO Advanced Research Workshop, Mazagon, 29 September-4 October 1991.

[5] Mersini-Houghton, L. and Vaas, R. (2012) The Arrows of Time: A Debate in Cosmology. Springer, Berlin. http://dx.doi.org/10.1007/978-3-642-23259-6

[6] Hawking, S.W. (1985) Physical Review D, 32, 2489. http://dx.doi.org/10.1103/PhysRevD.32.2489

[7] Kiefer, C. and Zeh, H.D. (1995) Physical Review D, 51, 4145. http://dx.doi.org/10.1103/PhysRevD.51.4145

[8] Joos, E. and Zeh, H.D. (1985) Zeitschrift für Physik B, 59, 223-243. http://dx.doi.org/10.1007/BF01725541

[9] Everett, H. (1957) Reviews of Modern Physics, 29, 454. http://dx.doi.org/10.1103/RevModPhys.29.454

[10] DeWitt, B.S. (1967) Physical Review, 160, 1113. http://dx.doi.org/10.1103/PhysRev.160.1113

[11] Hartle, J.B. and Hawking, S.W. (1983) Physical Review D, 28, 2960. http://dx.doi.org/10.1103/PhysRevD.28.2960

[12] Zeh, H.D. (1986) Physics Letters A, 116, 9-12. http://dx.doi.org/10.1016/0375-9601(86)90346-4

[13] Barbour, J. (2000) The End of Time. Orion, London.

[14] Barbour, J. The Nature of Time. arXiv:0903.3489 [gr-qc].

[15] Ellis, G.F.R. On the Flow of Time. arXiv:0812.0240 [gr-qc].

[16] Kiefer, C. Does Time Exist in Quantum Gravity? arXiv:0909.3767 [gr-qc].

[17] Rovelli, C. Forget Time. Foundations of Physics, 41, 1475-1490. arXiv:0903.3832 [gr-qc]. http://dx.doi.org/10.1007/s10701-011-9561-4

[18] Hawking, S.W. (1994) My Greatest Mistake. In: Halliwell, J.J., Perez-Mercader, J. and Zurek, W.H., Eds., Physical Origins of Time Asymmetry, Cambridge University Press, Cambridge, 346-358.

[19] Hartle, J.B. (2005) American Journal of Physics, 73, 101. http://dx.doi.org/10.1119/1.1783900

[20] Schulman, L.S. (2005) Entropy, 7, 221-233. http://dx.doi.org/10.3390/e7040221

[21] Maroney, O.J.E. (2010) Foundations of Physics, 40, 205-238. http://dx.doi.org/10.1007/s10701-009-9386-6

[22] Landauer, R. (1961) IBM Journal of Research and Development, 5, 183-191. http://dx.doi.org/10.1147/rd.53.0183

[23] Landauer, R. (1996) Physics Letters A, 217, 188-193. http://dx.doi.org/10.1016/0375-9601(96)00453-7

[24] Bérut, A., Arakelyan, A., Petrosyan, A., Ciliberto, S., Dillenschneider, R. and Lutz, E. (2012) Nature, 483, $187-189$. http://dx.doi.org/10.1038/nature10872

[25] Krause, W., Seidel, G., Schack, B. and Heinrich, F. (2001) Entropy Reduction in Memory and Thinking. In: Sommerfeld, E., Kampass, R. and Lachmann, T., Eds., Fechner Day 2001, Proceedings of the 17th Annual Meeting of the International Society for Psychophysics, Pabst, Lengerich, 87-92.

[26] Carroll, S. (2010) From Eternity to Here: The Quest for the Ultimate Theory of Time. Plume, New York.

[27] Nikolic, H. (1998) Causal, Psychological, and Electrodynamic Time Arrows as Consequences of the Thermodynamic Time Arrow. Physics/9812006.

[28] Mlodinow, L. and Brun, T.A. (2014) Physical Review E, 89, Article ID: 052102. http://dx.doi.org/10.1103/PhysRevE.89.052102

[29] Rovelli, C. (2014) Why Do We Remember the Past and Not the Future? The “Time Oriented Coarse Graining” Hypo- 
thesis. arXiv:1407.3384 [hep-th].

[30] Rovelli, C. (2015) Is Time’s Arrow Perspectival? arXiv:1505.01125 [physics.hist-ph]

[31] Tegmark, M. (2014) New Scientist, 222, 28-31. http://dx.doi.org/10.1016/S0262-4079(14)60731-4

[32] Fox, D. (2011) Scientific American, 305, 36-43. http://dx.doi.org/10.1038/scientificamerican0711-36

[33] Zeissner, S. (2014) Zukunftserinnerung. BSc Thesis, TU Dortmund, Dortmund. 